$\left(\mathrm{p}=0.03, \mathrm{r}^{2}=0.49\right)$ and higher NPI total scores $\left(\mathrm{p}=0.01, \mathrm{r}^{2}=0.63\right)$ (see Figures $\mathrm{S} 1 \mathrm{~A}$ and S1B, respectively; available as supplementary material attached to the electronic version of this letter at www.journals.cambridge.org/jid_IPG). Similarly, there were trend level correlations of IL- 6 release with worse ratings on the $\operatorname{CDR}\left(\mathrm{p}=0.08, \mathrm{r}^{2}=0.33\right)$ and MMSE $\left(p=0.05, r^{2}=0.39\right)$.

In this pilot project, we sought to replicate prior findings suggesting that IL-6 release from LPS-stimulated PBMCs is higher in AD than cognitively normal controls. We found increased IL6 release ratios in $\mathrm{AD}$, with trend level significance at the higher LPS concentration only. This is not surprising given the small sample; since the results are in the predicted direction they offer support for hypothesis 1 . We sought to extend prior results by adding an $\mathrm{AD}$-specific stimulus $\left(\mathrm{A}-\beta_{1-42}\right)$, in an attempt to more closely model the neuroinflammatory processes of $\mathrm{AD}$. Modest support was seen for hypothesis 2 with $\mathrm{A}-\beta_{1-42}$ increasing the IL-6 release ratio by a factor of 2 in $\mathrm{AD}$ patients but not controls, although this was not statistically significant; these results are in the direction predicted by hypothesis 2 .

Additionally we found a significant trend correlation between IL- 6 release ratio and several clinical variables, including verbal category fluency (animals), NPI, CDR and MMSE. A higher IL-6 release ratio was associated with worse illness severity (higher NPI total and CDR, lower MMSE and category fluency). This suggests that the IL-6 release ratio may have value as a marker of AD disease severity, especially severity of neuropsychiatric symptoms (NPS). The latter appears to be a unique finding, and may reflect the involvement of inflammatory mechanisms (peripheral and central) in the development of NPS.

This study is limited by small sample size, assessment of only one candidate cytokine, and limited capacity to assess multiple reaction conditions. The strengths of the study include

doi:10.1017/\$1041610208008077

\section{The relationship between annual predicted future population growth rates and elderly suicide rates}

Two paradoxical hypotheses - (i) that countries with low population growth rates or a decline in population growth rate will have high elderly dependency ratios leading to high elderly suicide rates, a well-characterized cohort of $\mathrm{AD}$ and control participants and the extension of prior results to neuropsychiatric and functional as well as cognitive findings.

These preliminary results suggest that IL-6 release from PBMCs may be a useful marker of disease severity and NPS severity in AD, thus meriting further study.

\section{References}

Benveniste, E. N., Nguyen, V. T. and O'Keefe, G. M. (2001). Immunological aspects of microglia: relevance to Alzheimer's disease. Neurochemistry International, 39, 381-391.

Miller, A. H. and Raison, C. L. (2006). Cytokines, p38 MAP kinase and the pathophysiology of depression. Neuropsychopharmacology, 31, 2089-2090.

Reale, M. et al. (2004). Treatment with an acetylcholinesterase inhibitor in Alzheimer patients modulates the expression and production of the pro-inflammatory and anti-inflammatory cytokines. Fournal of Neuroimmunology, 148, 162-171.

Rosenberg, P. B. (2005). Clinical aspects of inflammation in alzheimer's disease. International Review of Psychiatry, 17, 503-514.

AdAM Kaplin, ${ }^{1}$ Katherine A.L. Carroll, ${ }^{2}$ Jenn Cheng, ${ }^{3}$ Rameeza Allie, ${ }^{4}$ Constantine G. Lyketsos, 'Peter CAlabresi $^{4}$ and Paul B. Rosenberg ${ }^{1}$

${ }^{1}$ Division of Geriatric Psychiatry and Neuropsychiatry, Department of Psychiatry and Behavioral Sciences, Johns Hopkins University School of Medicine, Baltimore, U.S.A.

${ }^{2}$ University of Oxford, U.K.

${ }^{3}$ Johns Hopkins University School of Medicine, Baltimore, U.S.A.

${ }^{4}$ Division of Neuroimmunology, Department of Neurology, Johns Hopkins University School of Medicine, Baltimore, U.S.A.

Email: prosenb9@jhmi.edu and (ii) that countries with high population growth rates will have high elderly suicide rates because of Durkheim's hypothesis that the overall cohort size may influence suicide rates due to competition for scarce resources - were supported by a recent study (Shah, 2008). The relationship between average annual population growth rates and elderly suicide rates was shown to be curvilinear (U-shaped curve) fitting the quadratic equation $y=a+b x+c x^{2}$ (where $y$ is the elderly suicide rate, $x$ is the average 
annual population growth rate and $a, b$ and $c$ are constants) using an ecological study design.

Population growth rate is a function of life expectancy and birth rate. Thus, in countries with low average annual population growth rates or decline in average annual population growth rates, elderly dependency ratios may be high because of increased life expectancy and low birth rate. This, in turn, may result in increased elderly suicide rates. The average annual growth rate may begin to increase in some countries due to an increase in birth rates. This, in turn, may lead to a decline in the elderly dependency ratios. Moreover, in turn, this may result in a decline in elderly suicide rates. As the average annual population growth rate continues to increase, at a critical point the composite influence of increased life expectancy, increased elderly population size and the increase in the proportion of elderly in the general population on elderly suicide rates may become more prominent. This may, in part, reflect Durkheim's hypothesis that the overall cohort size may influence suicide rates due to competition for scarce resources (Shah and De, 1998).

The same curvilinear (U-shaped curve) relationship between elderly suicide rates and the average annual predicted future population growth rate was examined. Data on elderly suicide rates for males and females in the age-bands $65-74$ years and $75+$ years for each listed country were ascertained from the World Health Organization (WHO) website (www.who.int/whosis/mort/table1.cfm). The median (range) of the latest available year for data on elderly suicide rates was 2000 (1985-2003). Data on the average annual predicted future population growth rate until 2015 for each listed country were ascertained from the United Nations Development Programme (UNDP) website (www. hdr.undp.org/reports/global/2005/pdf/hdr05_HDI. pdf). Curve estimation regression models were used to examine the curvilnear relationship between elderly suicide rates and the average annual predicted population population growth fitting the quadratic equation $y=a+b x+c x^{2}$.

doi:10.1017/\$1041610208008119

\section{Cultural aspects of the patient-doctor relationship}

The patient-doctor relationship is central to medicine. This relationship has two fundamental components (Calman and McLean, 1984). The first is the doctor's care, skill and knowledge; the second is information-giving to help the individual
Full data sets for elderly suicide rates and the average annual predicted future population growth were available for 80 countries. Table S1 (available online at www.journals.cambridge.org/jid_IPG as supplementary material to the electronic version of this letter) illustrates the curve estimation regression models, whereby the relationships between suicide rates in both sexes in both elderly age-bands and the average annual predicted future population growth rates were curvilinear (U-shaped curve) and fitted the quadratic equation $y=a+b x+c x^{2}$; the significance level was at least at the 0.05 level.

How can the average annual predicted future population growth rates predict current suicide rates given that this population growth has not yet occurred? It is possible that this observed relationship was purely due to chance. Also, the average annual predicted future population growth rate may be a proxy measure for other correlates of elderly suicides or other variables may predict both elderly suicide rates and the average annual predicted future population growth rates (epiphenomena). The findings of this brief study illustrate that considerable caution and care are required in interpreting findings from cross-sectional ecological studies exploring potential etiological relationships.

\section{References}

Shah, A. K. (2008). The relationship between population growth and elderly suicide rates: a cross-national study. International Psychogeriatrics. E-published ahead of print. doi: $10.1017 /$ S1041610208007953.

Shah, A. K. and De, T. (1998). Suicide and the elderly. International fournal of Psychiatry in Clinical Practice, 2, 3-17.

\author{
AJit SHaH \\ Professor of Ageing, Ethnicity and Mental Health, \\ University of Central Lancashire, Preston, and \\ Consultant Psychiatrist, West London Mental Health \\ NHS Trust, London, U.K. \\ Email: ajit.shah@wlmht.nhs.uk
}

make decisions. Hence, communication and trust are essential in this relationship.

The problem arises when doctors see patients as sick people from whom to extract information or to whom to impart advice, and they therefore ignore a vital purpose of communication, which is to initiate and enhance the relationship with their patients (Persaud, 2005). Long and Jiwa (2004) found that in $25 \%$ of medical consultations, the chief concerns of patients had not been elicited and $40 \%$ of cancer 